\title{
Dynamische Gruppenarbeit
}

\section{Dezentrale Feinplanungsunterstützung durch einen virtuel- len Marktplatz}

\author{
Thilo Münstermann, Jens Völqkee, Paul Flacbskampf \\ Institut für Unternehmenskybernetik. e.V., RWTH Aachen
}

\section{Einleitung}

In deutschen Maschinenbauunternehmen ist Gruppenarbeit weit verbreitet. Untersuchungen zufolge fand in den 90er Jahren insbesondere bei Unternehmen mit mehr als hundert Beschäftigten eine Orientierung hin zur Arbeitsgruppenbildung an Bearbeitungsmaschinen statt. Die stärkste Ausprägung dieser Entwicklung war bei direkt-produktiven Tätigkeiten zu beobachten: Während im Jahr 1993 lediglich $40,4 \%$ der Beschäftigten in Gruppen zusammengearbeitet hat, traf dies im Jahr 1998 bereits für $46 \%$ der Beschäftigten zu (Sauerwein 2000).

Unter Gruppenarbeit wird im industriellen Umfeld die Zusammenarbeit mehrerer Menschen zur Erreichung einer gemeinsamen Zielsetzung verstanden. Die Gruppe übernimmt eigenverantwortlich eine ganzheitliche Aufgabe in einem räumlich zusammengefassten Arbeitssystem, und die Gruppenmitglieder verteilen die Teilaufgaben innerhalb des Arbeitssystems selbständig (Antoni et al. 2003). Diese Selbständigkeit geht mit einer Verlagerung von Planungsprozessen von der hierarchisch höheren, zentralen Planungsstelle zu den einzelnen Arbeitsgruppen einher (Dezentralisierung).

Die ursprüngliche Motivation für die Bildung von Arbeitsgruppen liegt in der Vermeidung von Monotonie des Arbeitsalltags. Durch Abwechslung und Partizipation der Mitarbeiter sollen die Motivation und damit die Produktivität gesteigert werden (Wiendahl 2005). Ein großes Potenzial der Gruppenarbeit liegt in der dezentral durchgeführten Feinplanung der Auftragsfertigung. Bestehende ERP/PPSLösungen bieten in diesem Bereich wenig bis gar keine Unterstützung für Gruppen mit wechselnder Zusammensetzung. Für diese Form der Arbeitsaufteilung fehlen daher Informationssysteme, mit denen Gruppen bei der operativen und gruppenübergreifenden Ressourcensteuerung und Auftragsfeinplanung unterstützt werden und dabei die leistungsgerechte Entlohnung berücksichtigen. Ein virtueller Markt- 
platz soll hier die bestehenden ERP/PPS-Systeme unterstützen und ergänzen. Mit der zusätzlichen Möglichkeit von dezentraler Feinplanung können bestehende Systeme effizienter und zeitsparender genutzt werden. In Praxisbeispielen konnte die Auftragsdurchlaufzeit dadurch um bis zu 32\% reduziert werden (Lay und Maloca 2005).

Eine weitere mögliche Motivation für die Bildung von autonomen Arbeitsgruppen in der Fertigung, liegt in der zu beobachtenden Steigerung der Komplexität und Dynamik der Unternehmensumwelt (Malik 2004). Die Unternehmen erkennen, dass Sie häufig durch die Einführung von starren Planungssystemen eher Flexibilität in der Fertigung verloren haben. Dieses Ungleichgewicht zwischen Innen- und Außenkomplexität schwächt die Unternehmen (Ashby 1956). Um zu überleben muss in den Unternehmen dieser Entwicklung entgegengesteuert werden.

Oft führt Gruppenarbeit in Betrieben aber nicht zum gewünschten Erfolg, da sie nach kurzer Zeit wieder „einschläft“ oder Gruppenzuteilungen oder Arbeitgruppen an sich ganz ignoriert werden (Fecht und Unbehend 2003). Grund dafür ist häufig die Vernachlässigung der aufwändigen und komplizierten Absprachen und Planungen. Ein Arbeitstool was hier ansetzt kann nicht nur die Effizienz der Planung und Gruppenarbeit erhöhen sondern auch dazu beitragen sie anwenderfreundlicher zu gestalten und Kommunikation und Planung aufrecht zu erhalten, um so den eingeführten Gruppenarbeitsprozess zu erhalten (Askri et al. 2008).

Um die Möglichkeiten der dezentralen Feinplanung durch Gruppenarbeit stärker ausschöpfen zu können, sind eventuelle besondere Rahmenbedingungen, insbesondere die begrenzten personellen und finanziellen Ressourcen der Unternehmen zu beachten. Die Reaktion auf variierende Auftragslagen und Abrufschwankungen erfordert beispielsweise dynamische Gruppen, deren Größe und Qualifikation der Mitglieder sich schnell verändern. Dazu gehören einfache und kostengünstige Möglichkeiten zur Messung und Kommunikation der Gruppenleistung sowie Konzepte zur Leistungsbewertung und Personaleinsatzplanung in den Gruppen bei häufigen Gruppenumbesetzungen. Letztere werden erforderlich, wenn z. B. selten eingesetztes Spezialwissen bei einzelnen Mitarbeitern vorhanden ist und in unterschiedlichen Gruppen benötigt wird.

Aus dem Bedarf nach Zugehörigkeit einzelner Mitarbeiter zu verschieden Gruppen entsteht eine weitere Anforderung. Die Leistung einzelner Facharbeiter in den Gruppen muss erfassbar sein und mit den Entgeltsystemen gekoppelt werden können. Anforderungen an zeitgemäße Entlohnungssysteme sind Transparenz und Leistungsbezogenheit, Motivierung, Gruppenarbeitsförderung, der wirtschaftliche Einsatz von Ressourcen und die Produktionsverbesserung (Heinz und Gosmann 1996).

Im Rahmen eines von der Arbeitsgemeinschaft industrieller Forschungsvereinigungen (AiF) aus Mitteln des BMWi geförderten Forschungsvorhabens entstand in Kooperation des Instituts für Unternehmenskybernetik e.V. und des Instituts für Integrierte Produktion Hannover gGmbH ein Konzept sowie eine beispielhafte 
Umsetzung eines solchen Informationssystems, im folgenden Arbeitsgruppenassistent (AGA) genannt. Der Fokus des vorliegenden Artikels liegt auf der Beschreibung eines virtuellen Marktplatzes zur Ressourcenverhandlung zwischen Gruppen. Um diese Idee in das Gesamtkonzept einordnen zu können, wird im Folgenden zunächst die grundlegende Funktionsweise kurz vorgestellt. Eine detaillierte Beschreibung des Feinplanungskonzeptes und der zugrundeliegenden Methoden findet sich bei Henning et al. (2009).

\section{Vorgehensweise und erreichte Ergebnisse}

$\mathrm{Zu}$ Beginn wurde eine IST-Analyse zum Stand der Gruppenarbeit, der Anforderungen an die Flexibilität und den bestehenden Software-Systemen in kleinen und mittelständischen Unternehmen der Metallverarbeitung durchgeführt und durch eine Technikfolgenabschätzung ergänzt (Flachskampf et al. 2008). Anschließend wurden die benötigten Methoden zur Messung von Zielgrößen, zur Erfassung von Restriktionen und zum Kapazitätsabgleich zwischen Gruppen theoretisch entwickelt (Henning et al. 2009, S. 20-34).

Die Implementierung und Realisierung des AGA erfolgte in einem Prozess der agilen Softwareentwicklung (Westerwick 2000). Dabei wurden beide Pilotanwender von Anfang an intensiv in die Entwicklung einbezogen. Die agile Softwareentwicklung ist durch mehrere Iterationszyklen gekennzeichnet. Jeder Zyklus besteht aus Befragung, Anforderungsanalyse, Umsetzung und Testen. Demzufolge wurde der AGA im Projekt iterativ und inkrementell entwickelt. Die Entwicklung des Softwaretools wurde in mehrere gleichartige Zwischenschritte aufgeteilt, die jeweils eine lauffähige Version mit gestiegener Gesamtfunktionalität zum Ergebnis hatten.

Die Anwendbarkeit der entwickelten Methoden wurde durch zwei Pilotanwender des Projektbegleitenden Ausschusses verifiziert. Um auch Unternehmensdaten verwenden zu können, wurde eine Verknüpfung zwischen dem AGA und vorhandenen Planungssoftwaresystemen implementiert. So konnten Daten, z. B. die Terminierung und die Grobplanung, als Grundlage für die Feinplanung genutzt werden.

Der AGA wurde abschließend in den Pilotunternehmen eingeführt. Da eine agile Vorgehensweise bei der Entwicklung des Demonstrators gewählt wurde und die Beteiligten von Anfang an stark involviert waren, war keine intensive Schulung der Administratoren und Mitarbeiter oder aufwendige Einführungsmaßnahmen notwendig. Die Erfahrungsergebnisse der Pilotanwender wurden für die Verbesserung der Methoden und des AGA genutzt. 


\section{Dynamische Gruppenwechsel durch einen virtuellen Marktplatz}

\section{1 Überblick}

Der AGA ist als Erweiterung zentraler Produktionsplanungssysteme konzipiert und entweder als eigenständige Software über Schnittstellen mit diesen koppelbar oder als Modul in bestehende Systeme zu integrieren. Zur gruppenbasierten Feinplanung bietet der AGA dezentrale Planungstabellen für einzelne Arbeitsgruppen. Diese werden zentral gespeichert und sind über eine Konfliktbehandlung miteinander vernetzt, so dass gruppenübergreifende Auftragsplanung möglich bleibt, obwohl jede Gruppe zunächst mit ihrer eigenen Feinplanungstabelle arbeitet.

Die Konzeption der Software unterscheidet Mitarbeiter, welche eine Liste von Qualifikationen besitzen können, und Maschinen, welche durch Funktionen charakterisiert sind. Einzelne Arbeitsschritte sind über die zur Bearbeitung erforderlichen Qualifikationen und Funktionen definiert. Im Laufe des Planungsprozesses werden ihnen Zeiträume und konkrete Mitarbeiter und Maschinen zugewiesen. So werden auftrags-, mitarbeiter- und maschinenzentrierte Planungsvisualisierungen ermöglicht (Henning et al. 2009, S. 71 f.). Ein zentrales Konzept der Feinplanungstabellen bilden dynamische Pools, welche für einen Auftragsschritt in einem Zeitraum nur solche Ressourcen aus der Gruppe anzeigen, welche die benötigte Qualifikation und Funktion aufweisen, sowie freie und verbuchte Ressourcen farblich unterscheiden.

\subsection{Idee des virtuellen Marktplatzes}

Eine Grundidee des AGA besteht in der Dezentralisierung und damit der Dynamisierung von Feinplanungsprozessen. Gruppen sollen eigenverantwortlich innerhalb bestimmter Grenzen ihre Planung optimieren können und aufgrund der höheren Übersichtlichkeit einer Gruppenplanung schnell und flexibel auf Änderungen reagieren können. Wie jede Dezentralisierung bringt dies aber auch den Nachteil mit sich, dass betriebsweite Potenziale der Zusammenarbeit zunächst nicht genutzt werden, da keine zentrale Planungsstelle die innerhalb der Feinplanung benötigten oder frei werdenden Ressourcen gruppenübergreifend zuweist. Eine solche zentrale Zuweisung wäre auf dem Detaillierungsgrad einer Feinplanung mit enormem Arbeitsaufwand verbunden und würde gleichzeitig dem Prinzip der Gruppenautonomie widersprechen.

Um dennoch Möglichkeiten zur Planungsoptimierung (z. B. Verkürzung der Durchlaufzeit oder Einhalten von Lieferterminen) nutzen zu können wird im Folgenden das Konzept des virtuellen Marktplatzes eingeführt, auf dem Gruppen benötigte und frei gewordene Ressourcen sowie Aufträge tauschen, verschieben und handeln können. 
Das Konzept des virtuellen Marktplatzes beruht auf der Idee, dass aus der Grobplanung eine vorläufige Zuweisung der Aufträge oder Arbeitsschritte auf die einzelnen Gruppen in der Fertigung vorgenommen wurde und diese Gruppen in ihrer Zusammenstellung und Personalstärke für die durchschnittliche Arbeitsbelastung optimiert sind. Aufgrund von Krankheiten, Maschinenausfällen, unvorhergesehenen Problemen oder zwischengeschobenen Aufträgen hoher Priorität kann es jedoch immer wieder vorkommen, dass von dieser Planung abgewichen werden muss. Nach der Grundidee des AGA werden diese Abweichungen in der dezentralen Feinplanung der Gruppen behandelt.

So können bei den einzelnen Gruppen Kapazitätsengpässe und -überschüsse auftreten, die weiteres Optimierungspotenzial bieten. Um dieses Potenzial zu nutzen soll den Gruppen die Möglichkeit geboten werden, zusätzliche Mitarbeiter oder Maschinen für bestimmte Zeiträume anzufragen oder Arbeitsschritte zur Bearbeitung an andere Gruppen anbieten und abgeben zu können. Abbildung 1 stellt das Prinzip dar und deutet die jeweilige Verhandlungsrichtung aus Sicht der Gruppe A an.

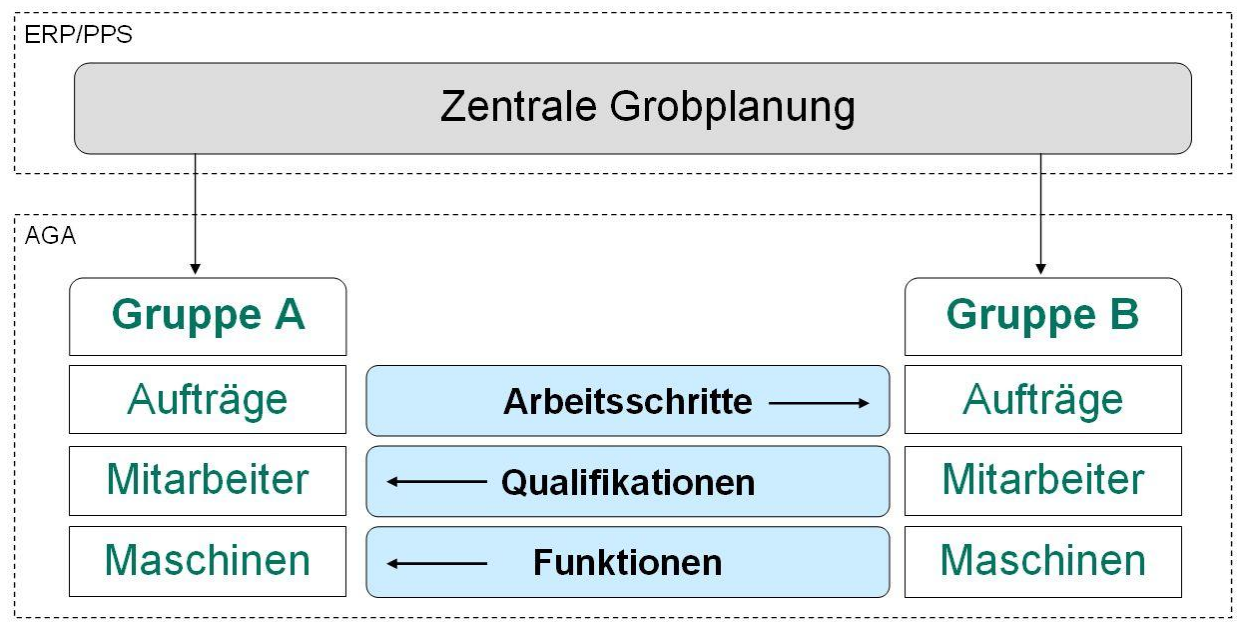

Abbildung 1: Verhandlungsebenen zwischen Gruppen (Henning et al. 2009)

Diese Verhandlungen können je nach Bedarf mehr oder weniger vollständig über den virtuellen Marktplatz vollzogen werden. In einer relativ kleinen Produktion mit wenigen Gruppen und Verantwortlichen wird die Nachfrage, ob man einen Mitarbeiter aus einer Gruppe für ein paar Stunden ausgeliehen haben kann, meist persönlich erfolgen. Bei einer größeren Produktion, besonders wenn diese über mehrere Hallen verteilt ist, kann die Anfrage nach einer benötigen Qualifikation für einen Zeitraum über den virtuellen Marktplatz gestellt, von anderen Gruppen beantwortet und schließlich beidseitig bestätigt werden. Die Abläufe zur Anfrage von Qualifikationen und Funktionen sowie dem Anbieten von Arbeitsschritten werden in den folgenden drei Kapiteln ausführlich beschrieben. Im daran anschließenden 
Kapitel wird eine weitere Ergänzungsmöglichkeit der Idee des virtuellen Marktplatzes, die Versteigerung von Aufträgen und Ressourcen, beschrieben.

\subsection{Mitarbeiter anfragen}

Gründe für den Bedarf einer Gruppe nach einem temporären Mitarbeiter können z. B. Krankheit oder Verzögerung in vorgelagerten Arbeitsschritten sein. Es stehen zwar die benötigten Arbeitsplätze und das Material zur Verfügung und alle Vorgängerarbeitschritte sind erledigt, aber es ist keiner oder zu wenige Mitarbeiter mit der benötigten Qualifikation anwesend. Dies kann sowohl kurzfristig und damit akut, aber auch mehrere Tage im Voraus ersichtlich auftreten. Oft wird nun innerhalb einer Gruppe vielleicht umgeplant, andere Tätigkeiten werden vorgezogen oder es wird ein Mitarbeiter eingesetzt, der diese Tätigkeit nur rudimentär beherrscht.

Über den virtuellen Marktplatz kann die Gruppe hingegen anfragen, ob eine andere Gruppe für einen Zeitraum t einen Mitarbeiter mit der Qualifikation Q entbehren kann. Es wird also eine Qualifikation für einen Zeitraum angefragt und nicht ein Mitarbeiter direkt. Prinzipiell kann eine Anfrage speziell an eine Gruppe gerichtet werden oder an alle Gruppen. Im letzteren Fall erreicht die Anfrage alle Gruppen, die einen Mitarbeiter mit dieser Qualifikation haben. Im ersteren Fall unterstützt der AGA falls gewünscht bei der Gruppenauswahl. Sind Qualifikation und Zeitraum ausgewählt, werden die Feinplanungen der anderen Gruppen überprüft, ob eine Gruppe tatsächlich einen Mitarbeiter mit dieser Qualifikation für den Zeitraum $\mathrm{t}$ noch nicht verplant hat. Werden mehrere oder keine Gruppen gefunden, die diese Qualifikation nach ihrer derzeitigen Feinplanung sofort zur Verfügung stellen könnten, wird nach der Methode zum Kapazitätsabgleich zwischen Gruppen die Flexibilität der Gruppen verglichen und entsprechend die Gruppe mit der höchsten Flexibilität ausgewählt (Henning et al. 2009, S. 32 ff.). Alternativ zu einem festen Zeitraum, kann auch eine Stundenzahl innerhalb eines Zeitraumes angefragt werden.

Bei der zweiten Variante ist die Chance auf eine Zusage höher, da einer ausleihenden Gruppe B mehr Flexibilität verbleibt. Auf eine Anfrage antwortet eine andere Gruppe immer entweder mit einer Ablehnung, oder mit einem konkreten Mitarbeiter und einem festen Zeitraum. Der Zeitraum kann sich dabei vom angefragten Zeitraum unterscheiden und somit ein Gegenangebot bilden. Wurde kein fester Zeitraum angefragt oder der Zeitraum von Gruppe B verändert zurückgeschickt, ist die Verhandlung erst durch eine erneute Bestätigung durch Gruppe A abgeschlossen. Auch wenn die Anfrage an mehrere Gruppen gesendet wurde, ist eine abschließende Bestätigung durch Gruppe A erforderlich. Andernfalls kann die Verhandlung bereits nach dem zweiten Schritt von Gruppe B abgeschlossen werden, wenn die Anfrage unverändert besteht.

Nach einer erfolgreichen Verhandlung einer Mitarbeiterausleihe werden die Rahmendaten in eine Ausleihtabelle eingetragen. Diese Tabelle enthält immer Zeit- 
räume, die minutengenau abgegrenzt sind, einen konkreten Mitarbeiter, eine verleihende und eine ausleihende Gruppe:

von dd.mm.yyyy hh.mm | bis dd.mm.yyyy hh.mm | [mitarbeiter] | von [gruppe] | an [gruppe]

Die Filter der dynamischen Pools und die Konfliktbehandlung der Feinplanung berücksichtigen diese Tabelle. Ausgeliehene Mitarbeiter werden in den Pools der Gruppe A für diesen Zeitraum mit eingeplant und können Arbeitsschritten, die innerhalb dieses Zeitraums liegen, zugewiesen werden. Die Konfliktbehandlung überprüft, ob Arbeitsschritte mit einem ausgeliehenen Mitarbeiter für Zeitraum t außerhalb dieses Zeitraumes verschoben werden und zeigt ggf. einen Konflikt an. Der Konflikt wird auch angezeigt, wenn der Mitarbeiter theoretisch nach der Feinplanung von Gruppe B Zeit hätte, um anzuzeigen, dass die im virtuellen Marktplatz getroffene Vereinbarung verletzt worden ist. In der mitarbeiterzentrierten Planung erscheint für den Ausleihzeitraum bei Gruppe A ein neuer Mitarbeiter, bei Gruppe B wird der Zeitraum für den Mitarbeiter grau hinterlegt.

Zur Kennzeichnung ausgeliehener Mitarbeiter kann eine zusätzliche farbliche Markierung konfiguriert werden, oder ein beliebiges Symbol als Grafikdatei im AGA eingebunden werden.

\subsection{Maschinen anfragen}

Gründe für den Bedarf einer Gruppe nach zusätzlichen Maschinen können z. B. Ausfälle oder ungeplante Wartungsarbeiten sein. Wenn genügend Arbeitsschritte zur Bearbeitung freigegeben sind und beispielsweise genug personelle Ressourcen zur Verfügung stehen, um mehrere Arbeitsschritte parallel erledigen zu können, macht es Sinn bei anderen Gruppen nach Maschinen bzw. Arbeitsplätzen zu fragen.

In diesem Fall kann eine Gruppe A über den virtuellen Marktplatz eine Funktion F für einen Zeitraum t bei einer Gruppe B anfragen. Auch bei dieser Anfrage kann ein fester Zeitraum oder eine Stundenzahl innerhalb eines Zeitraums für die Anfrage verwendet werden. Die Gruppenauswahl wird analog zur Anfrage von Mitarbeitern durch den AGA unterstützt, lediglich die Flexibilitätsbewertung von Gruppen entfällt, da diese im AGA lediglich für Qualifikationen stattfindet. Nach erfolgter Anfrage kann Gruppe B die Anfrage ablehnen, unverändert bestätigen oder ggf. den Zeitraum anpassen und als Gegenangebot zurück senden.

Nach einer erfolgreichen Verhandlung wird die Ausleihe einer Maschine in eine Ausleihtabelle wie folgt eingetragen:

von dd.mm.yyyy hh.mm | bis dd.mm.yyyy hh.mm | [maschine] | von [gruppe] | an [gruppe]

Auch diese Ausleihtabelle wird von der Feinplanung berücksichtigt. Ausgeliehene Maschinen werden im Maschinenpool der Gruppe A für diesen Zeitraum ange- 
zeigt und können Arbeitsschritten, die innerhalb dieses Zeitraums liegen, zugewiesen werden. Die Konfliktbehandlung überprüft, ob Arbeitsschritte mit einer ausgeliehenen Maschine für Zeitraum $\mathrm{t}$ außerhalb dieses Zeitraumes verschoben werden und zeigt ggf. einen Konflikt an. Auch dieser Konflikt wird unabhängig davon angezeigt, ob die Maschine tatsächlich für den Zeitraum verplant ist, sondern zeigt lediglich an, dass der Zeitraum der Ausleihe überschritten wurde. In der maschinenzentrierten Planung erscheint für den Ausleihzeitraum bei Gruppe A eine neue Maschine, bei Gruppe B wird der Zeitraum grau hinter legt. Zur Kennzeichnung ausgeliehener Maschinen kann eine zusätzliche farbliche Markierung konfiguriert werden, oder ein beliebiges Symbol als Grafikdatei in den Arbeitsgruppenassistenten eingebunden werden.

\subsection{Arbeitsschritte anbieten}

Als Alternative zum Anfragen von Mitarbeitern und Maschinen können Gruppen bei Kapazitätsengpässen auch Arbeitsschritte an andere Gruppen abgeben. Im Gegensatz zu den beiden zuvor beschriebenen Verhandlungsszenarien, bittet die Gruppe A dabei nicht um zusätzliche Ressourcen, sondern möchte Arbeitsschritte abgeben.

Während bei den zuvor beschriebenen Ausleihen stets eine abstrakte Qualifikation und Funktion angefragt wurde, wird in diesem Fall ein konkreter Arbeitsschritt angeboten. Es wird also kein Arbeitsschritttyp angeboten, sondern ein konkreter Arbeitsschritt eines Auftrags. Dies bietet in diesem Fall den Vorteil, dass spezifische Kommentare zu dem Arbeitsschritt hinterlegt sein können und bei Bedarf auch bereits eine Maschine oder ein Mitarbeiter zugewiesen sein können. Als Zeitbegrenzung werden ein frühester möglicher Starttermin und ein spätester möglicher Endtermin angegeben.

Die entstandene Anfrage wird an alle Gruppen gesendet, die theoretisch die im Arbeitsschritt geforderten Qualifikationen und Funktionen besitzen. Die Gruppe, welche als erstes die Anfrage bestätigt, übernimmt den Arbeitsschritt. Auch bei dieser Variante der Verhandlung kann ein Gegenangebot abgegeben werden, indem Start und/oder Endtermin angepasst werden. Dies ist z. B. sinnvoll, wenn Gruppe B den Auftrag zwar übernehmen könnte, aber nur dann, wenn sie schon ein paar Stunden früher als in der Anfrage angegeben, damit starten könnten. Gruppe A kann dann ihre Planung überprüfen, ob sie es schaffen alle Vorgängerschritte rechtzeitig fertig zu stellen. Die Verhandlung ist in diesem Fall erst abgeschlossen, wenn Gruppe A die Änderung des Zeitfensters akzeptiert und bestätigt. Nach einer erfolgreichen Verhandlung wird die Übernahme eines Arbeitsschrittes in eine Ausleihtabelle eingetragen.

Ausgeliehene Arbeitsschritte erscheinen anschließend in der Feinplanungstabelle der Zielgruppe und verschwinden aus der Feinplanung der ursprünglichen Gruppe. Die Konfliktbehandlung erkennt bei Verschieben des Arbeitsschrittes sowohl, ob der Auftragsschritt mit seinen Vorgängern oder Nachfolgern zeitlich überschnei- 
det, als auch, ob der verhandelte Zeitrahmen verlassen wird. Bei der maschinenund mitarbeiterzentrierten Planung erscheint der Arbeitsschritt wie alle anderen in der Planungstabelle. Zur Kennzeichnung ausgeliehener Arbeitsschritte kann eine zusätzliche farbliche Markierung konfiguriert werden, oder ein beliebiges Symbol als Grafikdatei in den Arbeitsgruppenassistenten eingebunden werden.

\subsection{Versteigerungen}

Über die oben beschriebenen Ausleihen hinaus, kann der virtuelle Marktplatz für Versteigerungen genutzt werden. Die Idee dahinter ist, dass zentral von der Unternehmensleitung oder der Produktionsplanung Aufträge den Gruppen angeboten werden. Dies ist als Ergänzung zu der festen Zuweisung von Aufträgen zu Arbeitsgruppen gedacht. Anstatt die Aufträge auf die Gruppen zu verteilen, kann auch ein Wettbewerb der Gruppen um die Aufträge gefördert werden. Im virtuellen Marktplatz werden dazu von zentraler Stelle neue Aufträge eingestellt. Die Aufträge enthalten bereits alle notwendigen Angaben, damit die Gruppenleiter sie in eine fiktive Planung integrieren können. Diese Kalkulation kann im AGA bereits vor der Zuteilung gemacht werden um die Abschätzungen realistischer zu machen.

Es werden zwei Arten von Versteigerungen unterschieden. Versteigerung nach Abgabetermin und Versteigerung nach niedrigstem Kostenvoranschlag. Die zweite Variante ist damit an ein monetäres Bonussystem gekoppelt, bei der die Gruppen für jeden übernommenen Auftrag einen bestimmten Bonusbetrag erhalten. Beide Arten der Versteigerung laufen so ab, dass die Gruppen bis zu einem vorher festgelegten Termin Angebote abgeben können. Nach Ablauf des Termins kann der AGA entweder automatisch den Auftrag an das beste Angebot (frühester Liefertermin oder niedrigster Geldbetrag) vergeben oder eine manuelle Zuteilung findet durch einen Entscheider statt.

Je nachdem wie die Gruppen in einem Unternehmen eingeteilt sind, macht die Versteigerung von ganzen Aufträgen an Gruppen keinen Sinn, z. B. wenn jeder Auftrag in der Regel von mehreren Gruppen bearbeitet werden muss. In diesem Fall können genau wie ganze Aufträge auch einzelne Auftragsschritte versteigert werden. Der koordinatorische Aufwand bei hoher Abhängigkeit der Schritte untereinander steigt jedoch, da sichergestellt werden muss, dass die zugesagten Termine zusammen passen und der Auftrag insgesamt termingerecht geliefert werden kann.

Wird in einem Unternehmen über Geldbeträge verhandelt verfügen die Gruppen über virtuelle Konten. Diese Konten können ebenfalls genutzt werden, um die oben beschriebenen Ausleihfunktionen für die Gruppen interessanter zu machen. Für einen Mitarbeiter oder eine Maschine kann ein Geldbetrag geboten werden, ebenso für das Übernehmen eines Arbeitsschrittes. 


\subsection{Zusammenfassung}

Der virtuelle Marktplatz bietet verschiedene Verhandlungsmöglichkeiten. Diese können, neben der individuellen dezentralen Feinplanung der einzelnen Gruppen, für eine unternehmensweite Optimierung der Produktion durch Austausch zwischen den Gruppen genutzt werden. Das Prinzip der virtuellen Verhandlungen stellt sicher, dass Auslastungen gleichmäßig über die Gruppen verteilt und Ressourcen möglichst gut genutzt werden. Ressourcen werden auf diese Weise nur dann abgegeben, wenn die Gruppe sie selber nicht braucht, oder es schafft ihre Planung entsprechend anzupassen. Die Flexibilität in der Fertigung kann durch den Marktplatz erhöht werden. Damit schafft sich das Unternehmen Freiräume, um angemessen auf Änderungen der Lage in der Fertigung reagieren zu können.

Prinzipiell ist davon auszugehen, dass die Nutzung des virtuellen Marktplatzes über finanzielle Anreize unterstützt werden muss (Flachskampf et al. 2008). Es muss sowohl für die Gruppe als auch für den Einzelnen mit Vorteilen verbunden sein, einen Mitarbeiter in eine andere Gruppe auszuleihen oder zusätzliche Auftragsschritte zu übernehmen. Über das im folgenden Kapitel kurz beschriebene Entgeltmodul können entsprechende Anreize geschaffen werden.

\section{Methode zur Entgeltfindung in Gruppen mit wechselnder Zusammensetzung}

\subsection{Grundidee der Methode}

Ziel des Entgeltsystems ist die Ermittlung eines Bonus-Punktwertes für jeden Mitarbeiter. Der in diesem Konzept vorgesehene Punktwert kann sowohl zur Verteilung eines ERA basierten Leistungsbonus, zur Verteilung eines tarifunabhängigen unternehmensweiten Bonusbetrags oder zur Ermittlung flexibler, individueller Zusatzzahlungen verwendet werden. Die Entscheidung darüber findet also bewusst nicht innerhalb dieser Methode, sondern bleibt der Personalabteilung oder sonstigen Entscheidern überlassen.

Die Methode basiert auf einer individuellen Entlohnung für einen Mitarbeiter, die er sich selbst erarbeiten kann, und einer Entlohnung für eine Gruppe. Dieser zweidimensionale Ansatz wird gewählt, um zum Einen der Einzelleistung eines jeden Mitarbeiters gerecht zu werden und zum Anderen den Leistungen innerhalb einer Gruppe Rechnung zu tragen (Bohnhoff 1991).

Die im Folgenden dargestellten Faktoren zur Entgeltberechnung stellen dabei eine Basismenge dar, aus der für jedes Unternehmen eine passende Auswahl getroffen werden kann. Abhängig von der Firmenpolitik und den strategischen Zielen kann so entschieden werden, was belohnt werden soll. Ist z. B. die Etablierung eines stärkeren Gruppengedankens notwendig, kann auf individuelle Leistungsentlohnung verzichtet werden. Gibt es hingegen eine sehr unterschiedliche Leistungs- 
bereitschaft unter den Mitarbeitern, kann eine stärkere Verlagerung auf individuelle Faktoren den notwendigen Anreiz zur persönlichen Einsatzsteigerung liefern. Zur Berechnung vieler Faktoren ist die Betrachtung eines Zeitraums $(t)$ notwendig, z. B. eines Monats oder Quartals.

\subsection{Entlohnungsfaktoren}

Im AGA sind elf Bausteine für den individuellen Entlohnungsteil umgesetzt worden. Jeder Baustein kann als ein Faktor zur Berechnung des Gesamtentgelts gesehen werden. Zahlreiche weitere Faktoren als die hier vorgestellten sind denkbar. Es wird in individuelle- und gruppenspezifische- Faktoren unterscheiden. $\mathrm{Zu}$ den individuellen Faktoren gehören:

Mitarbeiterqualifikation

Eingesetzte Mitarbeiterqualifikation

Häufigkeit der Springertätigkeit

Dauer der Springertätigkeit und

Geleistete Stunden außerhalb der normalen Arbeitszeit (Überstunden).

Die gruppenspezifischen Entlohnungsfaktoren bilden:

Mitarbeiterausleihstunden

Mitarbeiterqualifikationsausleihe

Maschinenausleihstunden

Maschinenfunktionsausleihe

Übernommene Auftragszeit und

Pünktliche Auftragsfertigstellung.

Die Bedeutung der Faktoren und ihre Berechnungsgrundlage auf Basis von Planungsdaten wird bei Henning et al (2009, S. 35 f.) detailliert beschrieben. Wichtig ist festzuhalten, dass eine gruppenübergreifende Abstimmung und Optimierung über einen virtuellen Marktplatz durch entsprechende (finanzielle) Anreize unterstützt werden muss. Ein entsprechendes Entgeltmodul muss stark parametrisierbar sein, um der Unternehmensführung zu ermöglichen, bestimmte Verhaltensweisen entsprechend den Unternehmenszielen und der -kultur zu fördern.

\section{Zusammenfassung und Ausblick}

Das Konzept des Arbeitsgruppenassistenten bietet Methoden und Softwarefunktionen an, um dezentrale Feinplanungsprozesse mit gruppenübergreifender $\mathrm{Zu}$ sammenarbeit und Optimierung zu unterstützen und diese mit Entgeltbonussystemen zu koppeln. Dabei wurden die einzelnen Möglichkeiten des AGA, zu denen Mitarbeiter anfragen, Maschinen anfragen, Arbeitsschritte anbieten und die Versteigerung zählen, sowie elf sinnvolle Faktoren der Entgeltberechnung beschrie- 
ben. Das Bonusentgeltsystem wurde dabei als umsetzungsnahste Variante beschrieben. Über eine frei zugängliche Demonstratorsoftware wurde die Umsetzbarkeit des Konzeptes gezeigt. Diese ist als Open Source Software unter http://sourceforge.net/projects/aga/ kostenlos zum Download bereit gestellt.

Weitere Möglichkeiten liegen zum einen in der Entwicklung automatischer Optimierungsverfahren, welche Potenziale der gruppenübergreifenden Zusammenarbeit erkennen und dabei die Planungsautonomie der Gruppen berücksichtigen. Henning et al. haben gezeigt, dass über eine Kombination aus dezentraler und zentraler Optimierung eine Reduktion der Komplexität von Produktionsplanungsoptimierung möglich ist (Henning et al. 2009, S. 122 ff.). Zum anderen muss das Konzept aufgegriffen und in bestehende ERP/PPS-Systeme integriert werden.

\section{Literatur}

Antoni C, Hofmann K, Bungard W (2003) Gruppen- und Teamarbeit: Neue Organisationsformen in Unternehmen. Ein Handbuch für das moderne Management. 2. Aufl. Springer Verlag, Berlin.

Ashby, W R (1956) An Introduction to Cybernetics. Chapman and Hall, London.

Askri A, Münstermann T, Flachskampf P (2008) Produktionsplanung durch dezentrale Feinplanungsschritte optimieren. In: phi - Produktionstechnik Hannover informiert, Ausgabe 11, April 2008 S. 10-11.

Bohnhoff A (1991) Ein prospektiv bewertetes Identifizierungssystem für schnell bewegte Güter in kombinierten Verkehr. VDI Verlag, Düsseldorf.

Fecht M, Unbehend M (2003) Gruppenarbeit in Produktionsbetrieben. Tectum Verlag, Marburg.

Flachskampf P, Münstermann T, Michulitz C, Askri A, Overmeyer L (2008) Einführung eines Softwaretools zur Unterstützung der Gruppenarbeit. Teil 5., In: Management-Kompetenz in kleinen und mittleren Unternehmen Jahrbuch der KMU Forschung und -Praxis. Hrsg. v. Meyer, Jörn-Axel: Lohmar - Köln: EUL Verlag, 319-329.

Heinz K, Gosmann R (1996) Entgeltgestaltung bei Gruppenarbeit - Erkenntnisse und Erfahrungen aus einem Arbeitskreis. REFA-Nachrichten Band 49 (1996) Heft 4, S. 26-29.

Henning K, Flachskampf P, Nickel R, Münstermann T (2009) Konzept für ein Informationssystem zur Unterstützung der Gruppenarbeit in mittelständischen Industriebetrieben der Metallverarbeitung. Books on Demand Verlag, Norderstedt. 
Lay G, Maloca S (2005) Aufgabenintegration - Abkehr vom Taylorismus? Stand der Nutzung integrierter Modernisierungskonzepte zur Ausweitung des Tätigkeitsspektrums auf Werkerebene, PI-Mitteilungen Nr. 36, Fraunhofer Institut für Systemtechnik und Innovationsforschung.

Malik, F. (2004) Systemisches Management, Evolution, Selbstorganisation. Grundprobleme, Funktionsmechanismen und Lösungsansätze für komplexe Systeme. 4. Auflage, Haupt Verlag, Bern, Stuttgart, Wien.

N.N. http://sourceforge.net/projects/aga. Abruf am 23.09.2009.

Saurwein R (2000) Zur Diffusion von Gruppenarbeit im Maschinenbau Gestaltung und Dynamik. In: Widmaier, U. (Hrsg.): Der deutsche Maschinenbau in den neunziger Jahren - Kontinuität und Wandel einer Branche, Campus- Verlag, Frankfurt 2000, S. 147-176.

Westerwick, A. (2000) Ein objekt- und nutzerorientierter SoftwareEntwicklungsprozeß am Beispiel eines arbeitsplatznahen Planungssystems in der Fertigung. VDI Verlag, Düsseldorf.

Wiendahl HP (2005) Betriebsorganisation für Ingenieure. Carl Hanser Verlag, München. 